\title{
Proteomics in Health and Disease
}

\author{
George L. Wright Jr* and O. John Semmes ${ }^{\dagger}$ \\ Department of Microbiology and Molecular Cell Biology, Virginia Prostate Center, \\ Eastern Virginia Medical School, Norfolk, VA 23501, USA
}

\begin{abstract}
One of the major goals of the postgenomic era is understanding the structures, interactions, and functions of all cell proteins. This becomes a daunting task considering the estimation that there are between 100000 and 200000 individual proteins resulting from alternative splicing of the 30000 genes encoded by the human genome. Since the cellular proteome is a dynamic profile, subject to change in response to various signals through posttranslational modification, translocation, and protein-protein and protein-nucleic acid interactions, the task becomes even more complex looming to a million or more modification events. Proteomics encompasses the study of expressed proteins, including identification and elucidation of the structure-function interrelationships which define healthy and disease conditions. Information at the level of the proteome is critical to understanding the function of cellular phenotype and its role in health and disease. Since posttranslational events and, indeed, an accurate assessment of protein expression levels cannot always be predicted by mRNA analysis, proteomics, used in concert with genomics, can provide a holistic understanding of the biology underlying the disease process. The challenge in deciphering the proteome is the development and integration of analytical instrumentation combined with bioinformatics that provide rapid, high-throughput, sensitive, and reproducible tools.
\end{abstract}

This issue of the Journal of Biomedicine and Biotechnology presents the first of a two-part series consisting of ten papers that describe both technical and bioinformatic advances to define the cell proteome towards a better understanding of health and disease. The current issue consists of the first five articles beginning with papers by Bensmail and Haoudi, and Pruess and Apweiler that describe bioinformatics approaches for defining the cancer cell proteome and for in silico proteomic analyses. Because of the high dimensionality of the data generated by proteomic methodologies, such as protein microarrays and mass spectral analyses, more efficient and accurate bioinformatics tools are required to mine and analyze the data. Major advances in mass spectrometry have resulted in rapid, high-throughput technologies for protein biomarker discovery, protein identification, disease analyses, and identification of posttranslational mod- ifications. One advance, SELDI ProteinChip mass spectrometry, is the subject of the next two papers that describe its use for biomarker discovery and its potential as a platform for development of multimarker clinical assays. The first paper by Reddy and Dalmasso presents a review describing the use of SELDI for biomarker discovery, drug discovery, protein identification, and for development of multiplex clinical assays, citing examples for cancer, neurological disorders, and infectious diseases. Feng and associates then describe an automated peak identification and calibration procedure for more precise mass analyses when attempting to differentiate disease from nondisease protein patterns. The last paper of this issue by Qoronfleh and associates describes a method for the isolation of membrane proteins for proteomic analysis.

The next issue (volume 2003, issue 5) presents the remaining five papers. This issue begins with a review by $\mathrm{Xu}$ and Lam on protein and chemical microarray approaches being utilized for proteomic studies. Then Flower and colleagues describe bioinformatics approaches for defining the immunome for discovery of novel vaccines. This is followed by a paper by Qoronfleh and colleagues who describe improved methods for detecting protein: protein interactions. Piccoli's research team then report a method for optimizing the rolling circle application technology for generating a sensitive high-throughput multiplex protein microarray for analysis of protein expression and molecular diagnosis. The final paper in this issue is by Vlahou and associates who describe the use of SELDI protein profiling coupled with a commercial decision tree learning algorithm for biomarker discovery and diagnosis of ovarian cancer.

The content of this special issue, although broad and addressing several key issues in proteomics research, still leaves many issues to be covered, especially functional and structural proteomics, in this fast evolving field of research. We anticipate addressing other new discoveries and applications in the proteomics field in future issues of the Journal of Biomedicine and Biotechnology.

George L. Wright Jr O. John Semmes 
George L. Wright Jr received the $\mathrm{PhD}$ degree from Michigan State University in 1966. His $\mathrm{PhD}$ research focused on deciphering the Mycobacterium proteome and detecting changes in the serum proteome of cows infected with tuberculosis using one- and twodimensional electrophoresis. He continued these studies first as a fellow and

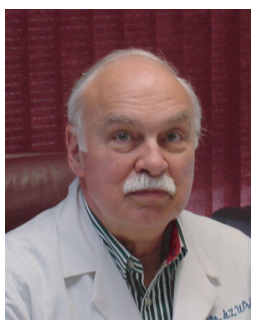
then as a faculty member at George Washington University from 1966 to 1973. Dr. Wright joined the faculty at Eastern Virginia Medical School (EVMS) in 1973, and became the department second Chairman in 1986. Since his arrival at EVMS, Dr. Wright's research has focused on the detection, identification, and characterization of biomarkers for early detection of urological cancers using a variety of molecular and proteomic technologies. In 1987, he cofounded the Virginia Prostate Center, a multidisciplinary program providing quality patient care, education, and research in urological diseases. The center's focus on proteomics resulted in the establishment of the Center for Biomedical Proteomics in 2001. Dr. Wright has published more than 150 original scientific papers and over 350 scientific presentations, and has 11 patents. He is a member of several scientific organizations, and has received numerous scientific awards; most recently (2000) the endowed EVMS Foundation Chair in Biomedical Science.

O. John Semmes received the $\mathrm{PhD}$ degree from George Washington University in 1989 in biochemistry. His PhD research addressed the structure/function of the high-affinity IL-2R. His postdoctoral studies at the National Institute of Health to investigate the molecular biology focused on the protein structure/function of human T-cell leukemia

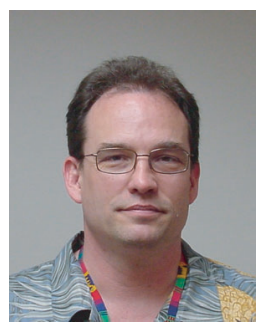
virus. Dr. Semmes joined the faculty at Johns Hopkins Medical School in 1995 as an Instructor. In 1997, he joined the University of Virginia as an Assistant Professor, and then as an Associate Professor at Eastern Virginia Medical School in 2000.

Dr. Semmes continues his research on HTLV-1utilizing both genomic and proteomic methodologies. He is currently the Director of the Center for Biomedical Proteomics, a program codeveloped with Dr. Wright, and is the Principal Investigator for the National Cancer Institutes' Early Detection Research Network Biomarker Discovery Laboratory at EVMS which focuses on biomarker discovery and early detection of prostate and breast cancers. He has published over 42 peer-reviewed scientific papers, given more than 150 scientific presentations, and had 4 pending patent applications. Dr. Semmes is a member of the HuPo Plasma group and a regular member of various NIH study sections and advisory groups concerned with cancer biology and proteomics.

\footnotetext{
* E-mail: wrightgl@evms .edu

$\dagger$ E-mail: semmesoj@evms .edu
} 

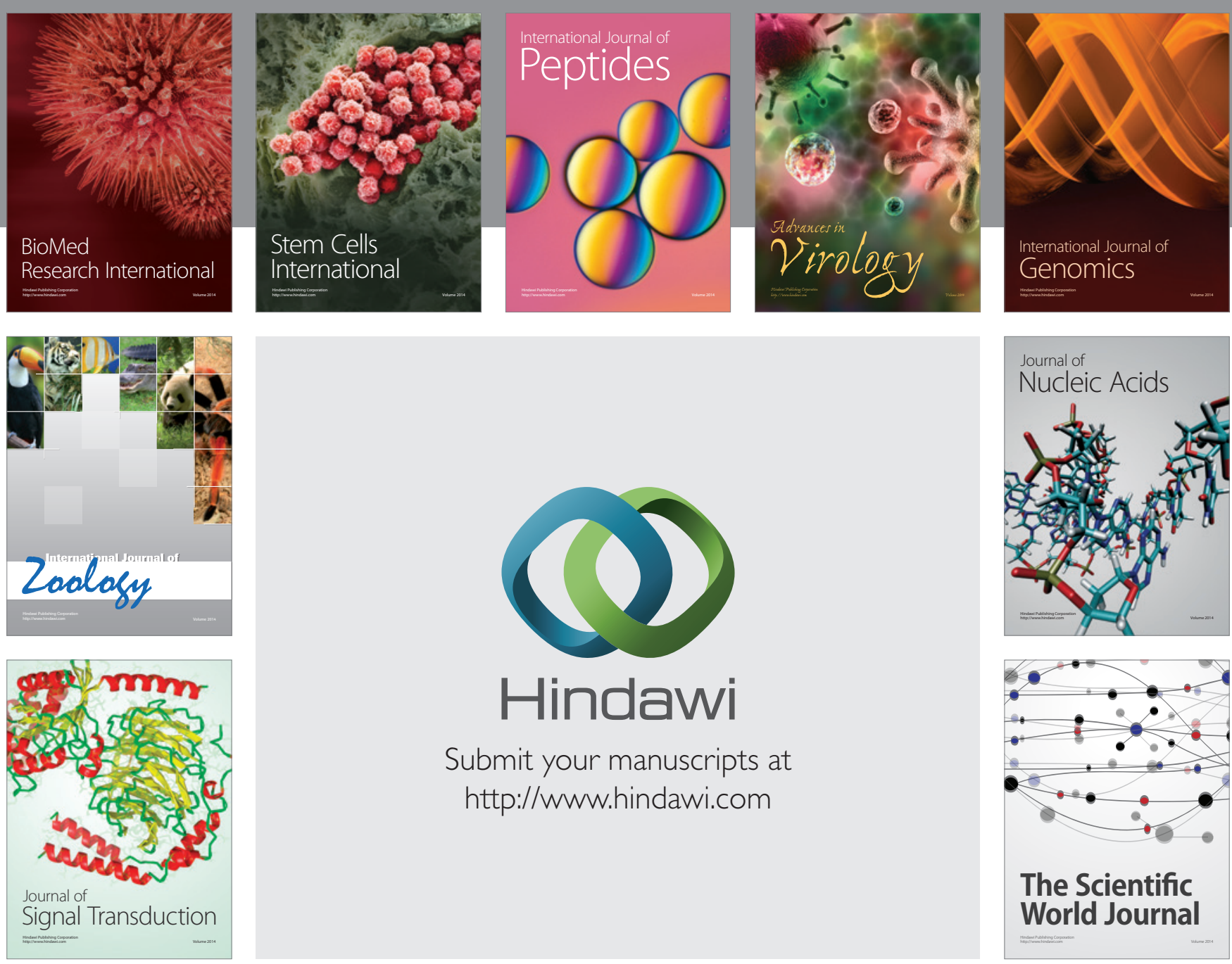

Submit your manuscripts at

http://www.hindawi.com
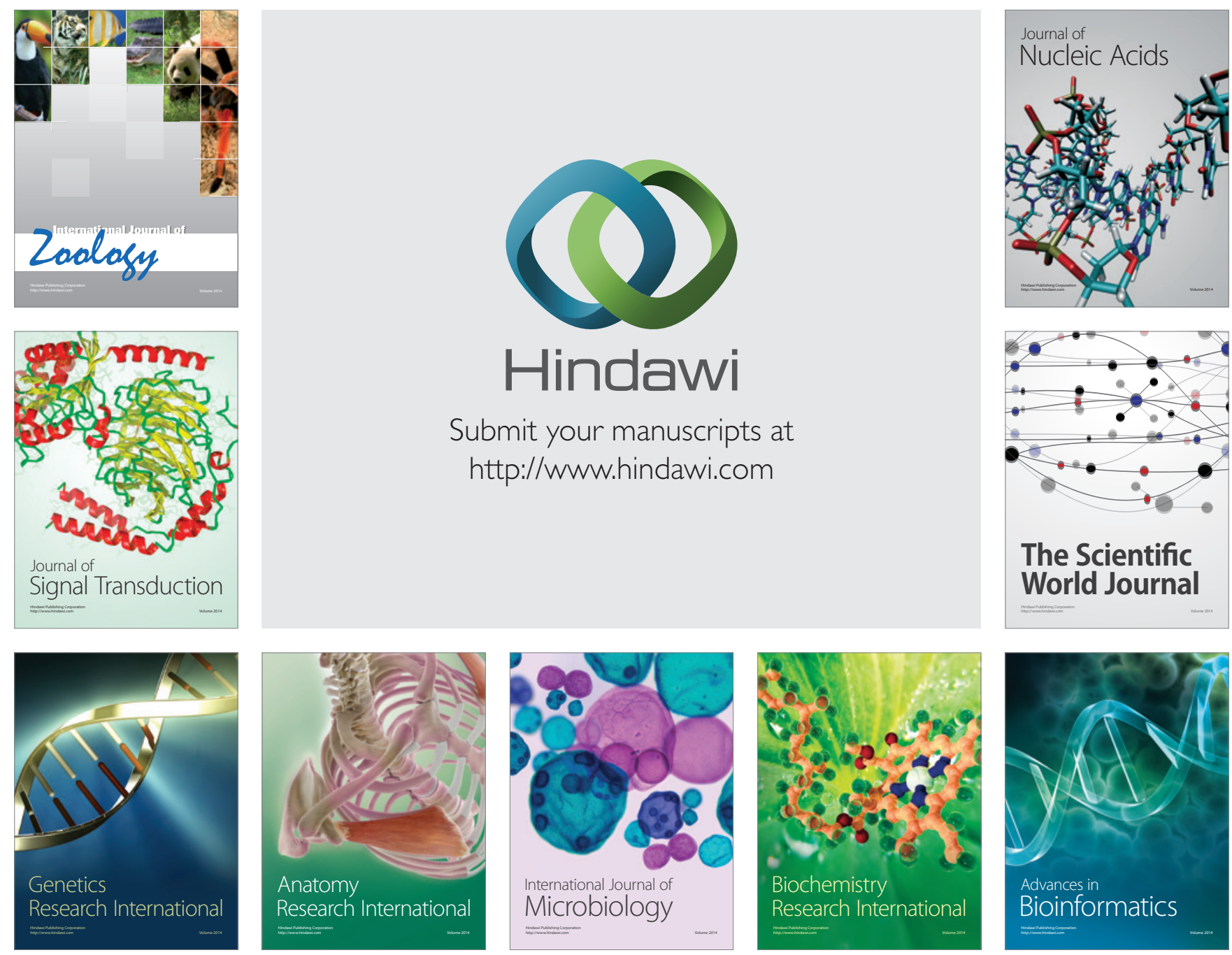

The Scientific World Journal
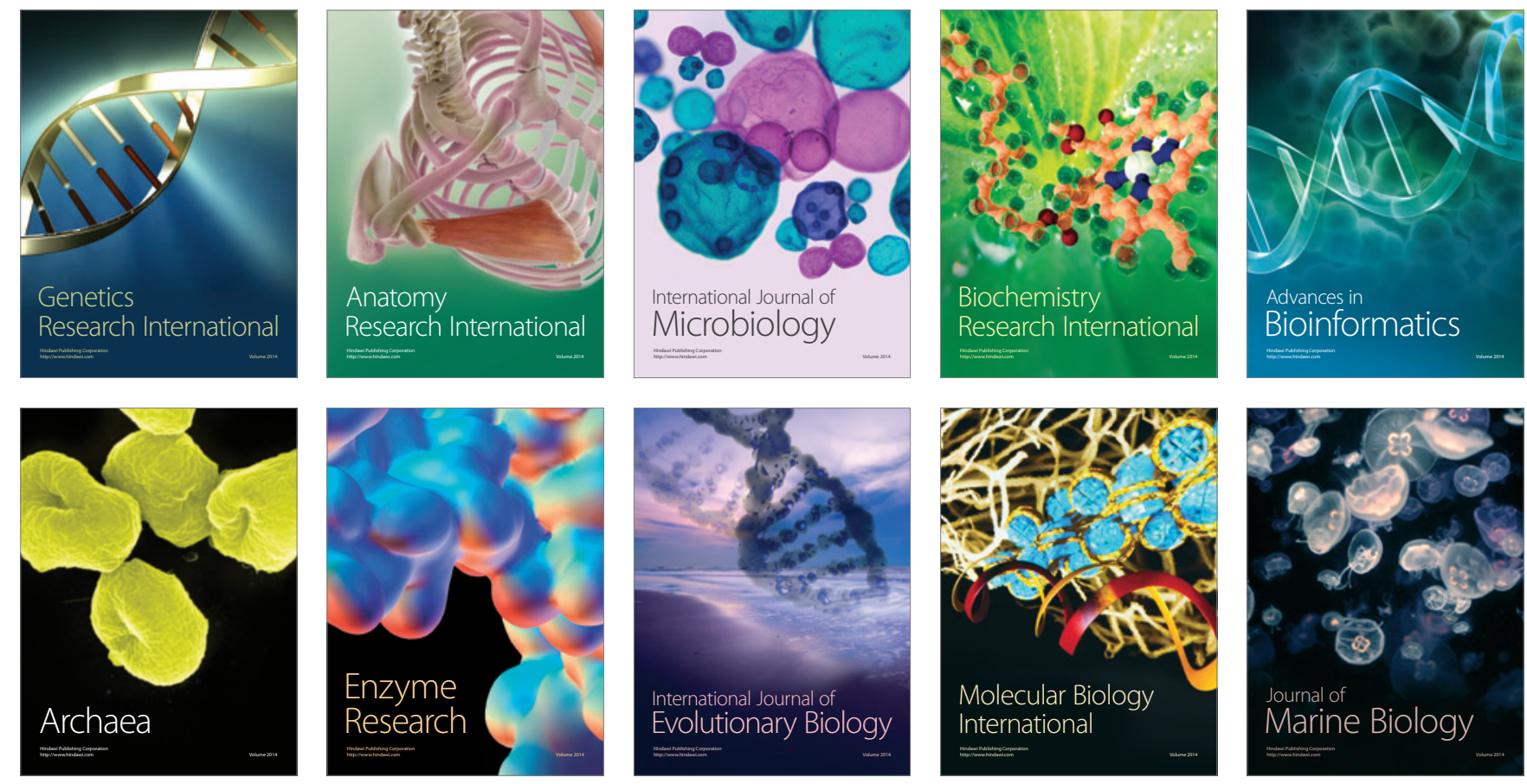